\title{
Genome-wide identification of miRNAs targets involved in cold response in
}

\section{cassava}

\author{
Shuxia $\mathrm{Li}^{1+*}$, Zhihao Cheng ${ }^{2+}$, and Ming Peng ${ }^{{ }^{*}}$ \\ ${ }^{1}$ Institute of Tropical Bioscience and Biotechnology, Chinese Academy of Tropical Agricultural Sciences, Haikou, \\ China \\ ${ }^{2}$ Haikou Experimental Station, Chinese Academy of Tropical Agricultural Sciences, Haikou, China \\ *Corresponding authors: Shuxia Li: lishuxia@itbb.org.cn; Ming Peng: pengming@itbb.org.cn. \\ ${ }^{\dagger}$ These authors contributed equally to this work.
}

\section{Abstract}

MicroRNAs (miRNAs) are recognized as essential transcriptional or post-transcriptional regulators, and play versatile roles in plants growth, development and stress responses. Cassava (Manihot esculenta) is a major root crop widely grown worldwide. Cold stress seriously affects cassava plants growth, development and yield. MiRNAs and their targets have been extensively studied in model plants, but a genome-wide identification of miRNAs' targets is still lacking in cassava. In this study, two degradome libraries were constructed using cold-treated and control cassava seedlings to identify the roles of miRNAs and their targets in response to cold stress. Following high-throughput sequencing and comparing with miRNA database, degradome data allowed us to identify a total of 151 non-redundant miRNA-target pairs. We revealed that 42\% of miRNA targets are conserved across plant species. However, 83 novel miRNA targets were identified in the two libraries. Gene ontology analyses showed that many target genes involved in cellular and metabolic process. In addition, 12 miRNAs and 31 corresponding targets of them were further found to be involved in cold stress response. Particularly, miR159, 164 and 396 participated in cold stress response by up-regulating certain transcription factors that were involved in the regulation of downstream gene expression. The work helps identifing cold-responsive miRNA targets in cassava and increases the number of novel targets involved in cold stress response. Furthermore, the findings of this study might provide valuable reference and new insights for understanding the functions of miRNA in stress response in plants.

Keywords: miRNA, Target genes, Degradome sequencing, qRT-PCR, cassava.

Abbreviations: miRNAs_microRNAs; GO_Gene Ontology; qRT-PCR_quantitative reverse transcription polymerase chain reaction; TFs_Transcription Factors.

\section{Introduction}

Cassava (Manihot esculenta) is a major root crop widely grown as a staple food, animal feed, and also as an important source of bioethanol in the tropical and subtropical regions of Latin America, Africa and Asia (E1-Sharkawy, 2004). It displays a unique ability to produce acceptable yields on marginal soils with minimal inputs and tolerate drought conditions. Nevertheless, as a tropical plant, cassava is categorized as a cold-sensitive species (Huang et al., 2005). Cold stress has been recognized as the most important limiting factor for its geographical location and productivity. Low temperatures and frozen conditions generally cause serious damage to cassava plants, such as reduced leaf expansion, chlorosis in leaves and yield decrease (An et al., 2012). In addition, the physiological status of cold-stressed cassava plants is also altered, such as transient increases in soluble sugars and proline levels, changes in membrane lipid composition, and increases in the level of reactive oxygen species (ROS) (An et al., 2012; An et al., 2016).

Thus, to stabilize cassava yield under unfavorable temperature stresses, it is necessary to identify key genes or pathways that can be used to improve cold stress tolerance of cassava via genetic engineering. So far, the mechanisms of cold stress response have been preliminarily investigated in the cassava. A multitude of putative cold stress tolerance-related genes have been identified and characterized by a combination of genetic, biochemical and high-throughput sequencing approaches (An et al., 2012; Zeng et al., 2014; An et al., 2016; Li et al., 2017a; Li et al., 2017b; Zeng et al., 2017). For examples, transgenic cassava plants that overexpressed Arabidopsis $C$-repeat-binding factors (CBFs) gene have enhanced tolerance to multiple abiotic stresses (An et al., 2017). Overexpression of two ROS-scavenging enzymes, cytosolic superoxide dismutase $(S O D)$ and ascorbate peroxidase (APX) led to increased tolerance to oxidative and chilling stresses in cassava (Xu et al., 2013). These studies advanced our knowledge regarding the molecular genetic mechanisms underlying the cold stress response in cassava.

MicroRNAs (miRNAs) are a class of 20-24 nucleotide (nt) endogenous noncoding RNAs that play essential roles in regulating gene expression in animals, plants, and fungi (Zhang 
et al., 2006). Plant miRNAs play regulatory roles at the post-transcriptional level by directly degrading target messenger RNAs (mRNAs) or repressing translation (Zhang et al., 2006; Rogers and Chen, 2013). In plants, miRNAs control the expression of genes encoding transcription factors, enzymes, and other important proteins, participating in the regulation of a wide range of biological processes such as normal growth, development, hormone signaling, nutrient homeostasis, and responses to various abiotic and biotic stresses (Hake, 2003; Eckardt, 2012; Shriram et al., 2016; Samad et al., 2017). In the past decade, a large number of miRNAs and targets have been identified across several plant species (Jones-Rhoades and Bartel, 2004; Eldem et al., 2012; Yadav et al., 2016; Feng et al., 2017). For instance, miR156 regulates expression of MYB genes involved in the control of many aspects of plant development, such as flowering under short days, primary root growth and seed germination (Reyes and Chua, 2007; Li et al., 2013a; Xue et al., 2017). Moreover, miR164 targets several different NAC (NAM/ATAF/CUC) genes to regulate the formation of shoot meristems, age-dependent leaf senescence and the specification of organ boundaries (Raman et al., 2008; Koyama et al., 2010; Li et al., 2013b). In the last decade, accumulating evidences place miRNAs and their targets in a central position within gene expression programs that underlie plant development and stress responses (Ori et al., 2007; Thiebaut et al., 2012; Zhou et al., 2013; Bresso et al., 2018). Numerous studies have shown that plant miRNAs are involved in the regulation of cold response in plants, such as Arabidopsis, rice, maize, barley, wheat, cotton, and barrel medic. For examples, eighteen cold-responsive miRNAs were identified in rice, such as miRNA166, 169 and 319 (Lv et al., 2010). Overexpression of miR319 or genetically down-regulating the expression of either of the two miR319-targeted genes, OsPCF5 and OsPCF8, led to enhanced cold tolerance in rice (Yang et al., 2013).

Given the important role of miRNAs in plant stress response, we speculated that miRNA and their targets might remarkably contribute to the cold stress tolerance of cassava. To date, approximately 48 miRNAs associated with cold stress were identified by establishing small RNA libraries of cassava (Zeng et al., 2010). However, a genome-wide identification of miRNAs' targets is still lacking. Further, we aimed to determine which miRNAs' targets and corresponding pathways responded to cold stress to facilitate survival of cassava. To address this question, we performed high-throughput sequencing and quantitative reverse transcription polymerase chain reaction ( $q R T-P C R$ ) to identify the conserved and cold-responsive miRNAs' targets in cassava leaves. A total of 151 miRNA-target pairs were identified using degradome sequencing and bioinformatics. Several conserved miRNAs' targets and the responses of these genes to cold stress were further discussed. Among them, 3 cold-responsive miRNA and corresponding targets were identified by qRT-PCR analyses. The results of this study provide new insights for understanding the miRNA-mediated regulatory network associated with cold stress in cassava.

\section{Results}

\section{Construction of the degradome library and sequencing analysis}

In order to identify the miRNA targets in cassava plants at a global level, two degradome libraries (Cold and Control library), which captured the cleaved mRNAs, were constructed for sequencing by a Hiseq2000 platform. A total of 24,256,967 and $24,655,669$ raw tags were obtained from Control and cold samples, respectively. After filtering the low-quality tags, the total number of clean tags in each library ranged from $24,256,875$ to $24,655,584$ tags with a mean value of $99.9 \%$. More than $98.5 \%$ of the clean reads were successfully mapped to the cassava reference genome to identify the fragments of degraded mRNAs (Table 1). Moreover, approximately $34 \%$ clean reads were uniquely mapped to the genome. These data indicated that our two degradome libraries were of high quality and recovered most of the degraded mRNA targets that contained the sequence profile of miRNA-mediated cleavage and allowed us to conduct further analysis.

\section{Systematic identification of miRNA targets}

In plants, the majority of miRNA-guided post-transcriptional regulation cleaves the target mRNAs between the 10th and 11th nt from the $5^{\prime}$ of miRNA in the complementary region of the miRNA:mRNA pair. In cassava, conserved miRNA targets were previously investigated mainly by bioinformatics prediction and only a few miRNA targets have been experimentally validated (Zeng et al., 2010; Chen et al., 2015). To better understand the functional roles of miRNAs, high-throughput degradome sequencing was performed in this study to identify more miRNA targets in cassava, particularly specific targets of cold-responsive miRNA targets. A total of 33,028 known transcripts and 176 miRNA sequences of cassava were used to annotate the degradome tags, and 144 target genes of 20 conserved miRNA families were verified (Supplementary Table S1). Based on the relative abundances of target site reads compared with other sites in the gene model, the miRNA targets were categorized into five classes (categories 0, 1, 2, 3 and 4). Under control condition, there were 83, 5, 32, 2 and 0 targets in categories $0,1,2,3$ and 4, respectively. Under cold condition, 88, 8, 20, 4 and 36 targets were grouped into categories $0,1,2,3$ and 4 , respectively (Supplementary Table S1). In total, the majority of targets were grouped into categories of greater than the median confidence (categories 3 and 4) in our degradome sequencing, accounting for $75 \%$. Finally, a total of 151 miRNA-target pairs, related to 20 miRNAs and 144 genes, were identified with a high confidence level ( $p$ value $<0.05$ ) (Supplementary Table S1). Among the 20 identified conserved miRNA families, MIR156, MIR 166, MIR 172 and MIR396 targeted multiple genes, whereas no targets were discovered for 16 miRNA families, such as MIR390, MIR397, MIR828, MIR1711, MIR2111, MIR1446 and MIR3627. The identified targets of the conserved miRNAs included many previously identified targets, such as the TCP genes for miR319, SQUAMOSA promoter binding protein-like $(S P L)$ genes as the targets of miR156, auxin response factor (ARF) as the target of miR167, and growth-regulating factors (GRFs) as the target of miR396 (Table 2). However, we also identified 83 novel target genes of miRNAs, including a lysine-specific histone demethylase (Manes.11G098200) for miR156, GTPase-activating protein for miR159, and a hypothetical protein (Manes.04G138500) as the target of miR396 (Supplementary Table S1). Many of the target genes of the conserved miRNAs were classified as the transcription factors (TFs), such as SPLS, AP2s, NACS, ARFs, MYBs, HD-ZIPs and GRFs, and so on. These TFs are known to 
regulate diverse aspects of plant growth, development, and biotic and abiotic stresses. Compared with model plants, such as Arabidopsis and rice, many conserved miRNA targets were found to be conserved in cassava, indicating that the miRNA-target relationship was evolutionarily conserved.

\section{GO analysis of miRNA target genes}

To classify the functions of the identified target genes for miRNAs, we performed Gene Ontology (GO) functional classification analysis. Based to GO analysis, a total of 144 miRNA targets were classified into three categories: biological process (BP), cellular component (CC) and molecular function (MF), which were classified into 15, 13 and 9 terms respectively, as shown in Figure 1 . Among these groups, the most significantly enriched GO terms were involved in the three main categories, "cellular process", "cell" and "binding", followed by "metabolic process", "cell part" and "catalytic binding". The results suggested that most target genes were associated with gene expression regulation and metabolic pathways.

\section{Identification of miRNA targets in response to cold stress}

In previous studies, we reported transcriptome changes in cassava in response to cold and identified over 6000 cold-responsive genes by analyzing the ssRNA-Seq data ( $\mathrm{Li}$ et al., 2017a). To further investigate the expression pattern of miRNAs-targeted genes under cold stress, we compared the expression values of the identified targets of control samples and those of cold-treated samples in the ssRNA-Seq data. According to the criteria described previously, 31 miRNAs targets were determined as the differentially expressed genes in cassava under cold stress (Table 3). Among them, 23 miRNAs targets were identified to be up-regulated and 8 were down-regulated by cold treatment. As shown in Table 3, five miR164-targeted NAC family genes, including Manes.06G090500 and Manes.14G080400, were strongly up-regulated by cold stress. This result is consistent with the expression pattern of miR164, which expression level was sharply declined to the lowest level at $24 \mathrm{~h}$ after cold treatment. MiR396 targeted a number of genes annotated as GRFs and NBS-LRR resistance proteins, which was also induced by cold stress. Altogether, these results indicated that the miR164, miR396 and family and their target genes played a key important function in responding to cold stress in cassava.

\section{Detection of the expression of miRNA and their targets by qRT-PCR}

To further reveal the dynamic expression pattern of miRNAs and their target genes, the expression profiles of 5 miRNA-mRNA pairs (miR159-MYB, miR164-NACs and miR396-GRFs) were selected, and qRT-PCR analyses were conducted to validate the alterations in their expression levels under cold conditions revealed by the high-throughput sequencing results. As shown in Figure 2, the expression levels of miR159 and miR396 decreased to the lowest value after cold treatment. However, within the same time period, their corresponding targets MYB and GRFs exhibited apparently the opposite expression profile. The expression correlation of these miRNAs and their targets demonstrated that they negatively regulated their targets. We also found that the expression level of miR164 and NACs genes were surprisingly induced by cold treatment. Further studies will be needed to elucidate the molecular function of these miRNAs and their targets in response to cold stress in cassava.

\section{Discussion}

MiRNAs play essential roles in response to abiotic stress, especially in cold stress response. High throughput sequencing and computational approaches have been used in recent years for identifying a huge number of miRNAs and targets in a range of plant species (Eldem et al., 2012; Geng et al., 2014; Yadav et al., 2016; Zhao et al., 2016; Feng et al., 2017; Zhang et al., 2017 Guo et al., 2018; Sahu et al., 2018; Zhang et al., 2018). However, in cassava, miRNA targets were previously investigated mainly via bioinformatics prediction, and only a few conserved miRNA targets have been experimentally validated (Zeng et al., 2010; Ballen-Taborda et al., 2013; Chen et al., 2015). Therefore, it is necessary to globally identify miRNA targets for interpretation of their functions. In this study, we constructed and sequenced two distinct degradome libraries using cassava leaves under normal and cold stress conditions. By analyzing degradome-Seq data, a large number of miRNA targets were successfully identified. These target transcripts generated significant $\mathrm{GO}$ terms related to binding and catalytic activities. Importantly, our degradome data verified $90 \%$ of previously validated targets. Additionally, our analysis revealed that the majority of these target genes were conserved among species and were obviously enriched in TFs and transcription regulatory activity, such as SPLS, MYBs, $H D$-ZIPS, ARFs, GRFs and TCP. This phenomenon was similar to that found in other plant species (Willmann and Poethig, 2007; Eldem et al., 2012; Geng et al., 2014; Feng et al., 2017).

In this study, several targets of conserved miRNAs such as the SPL gene (target of miR156) and TCP gene (target of miR319) have been reported that are required for abiotic stresses responses in some other plant species (Yang et al., 2013; Zhou et al., 2013; Cui et al., 2014), indicating the miRNAs and its targets may have a similar role in different plant species. In a previous study, eight conserved miRNAs (miR156, miR159, miR160, miR162, miR166, miR171, miR395, and miR396) were down-regulated under cold stress, while miR157, miR1168, and miR398 were up-regulated, and the corresponding targets were identified in our degradome sequencing (Zeng et al., 2010). The gene encoding the MYB domain transcription factor is a conserved target of the miR159 family in plants. In Arabidopsis, miR159 have been verified to target encoding MYBs to regulate plant growth and abiotic stresses responses (Li et al., 2016). In addition, MYB33 and MYB101 are targeted by miR159 and appear to play an important role in the response to abscisic acid (ABA) during seedling stress responses (Reyes and Chua, 2007). In this study, we detected a number of MYB family TFs regulated by mes-miR159. MiR159 expression is down-regulated after cold treatment, and regulates the abundance of MYB mRNAs in response to cold stress. MiR164 is a conserved miRNA in many plant species and guide the cleavage of the mRNAs of five NAC transcription factor genes in Arabidopsis that are involved in lateral root emergence (Guo et al., 2005; Li et al., 2012), boundary establishment (Laufs et al., 2004), and age-dependent cell death (Kim et al., 2009). Several studies have shown that miR164 is also involved in response to abiotic and biotic stress in plants (Lu et al., 2008; Zhao et al., 2012; Fang et al., 2014). 
Table 1. Data set summary of sequencing of the two degradome libraries.

\begin{tabular}{lll}
\hline Type & Control & Cold \\
\hline Raw tags & $24,256,967$ & $24,655,669$ \\
Clean tags & $24,256,875(99.9 \%)$ & $24,655,584(99.9 \%)$ \\
Total mapped tags & $23,792,501(98.1 \%)$ & $24,291,679(98.5 \%)$ \\
Unique mappable tags & $8,002,069(33.6 \%)$ & $8,595,344(35.3 \%)$ \\
cDNA mapped tags & $11,420,405(48.9 \%)$ & $10,202,506(42.6 \%)$ \\
\hline
\end{tabular}

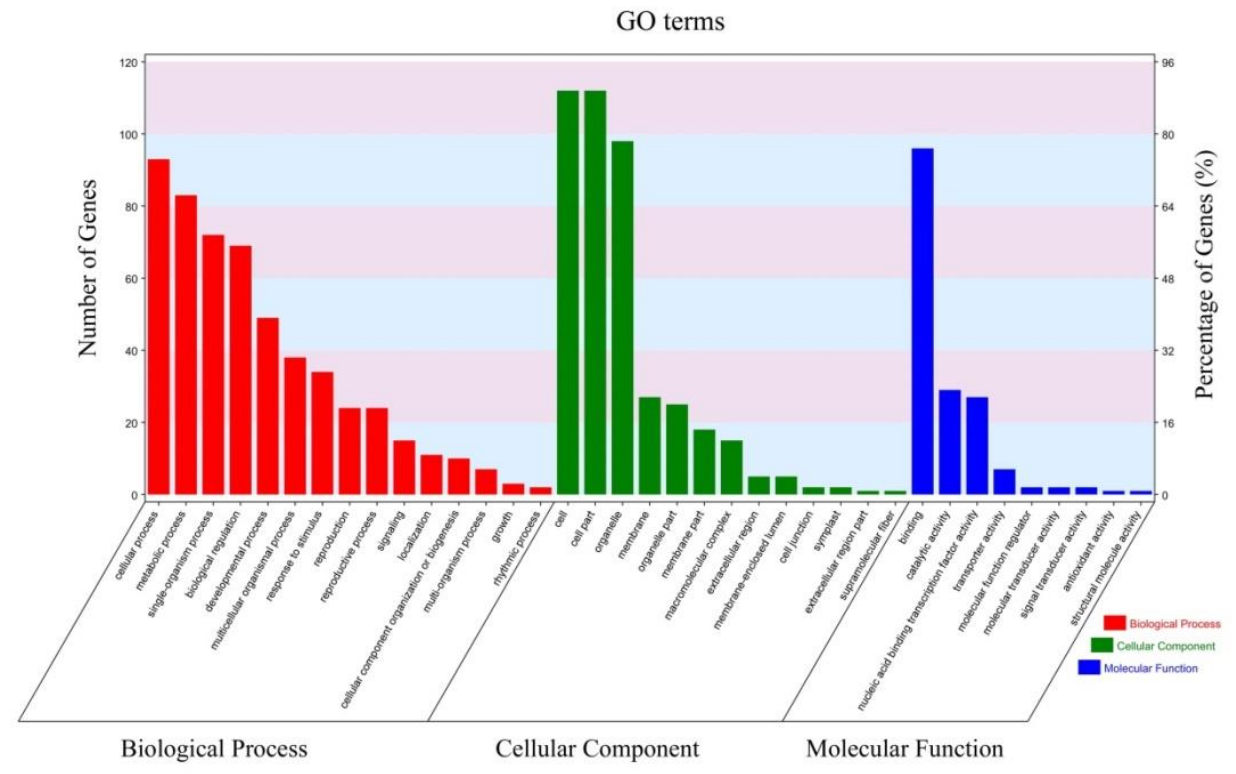

Fig 1. Gene Ontology functional classification of the miRNA targets identified in cassava. The $x$-axis represents the diverse biological functions of the targets according to three GO categories (biological process, cellular component and molecular function). The $y$-axis represents the number of the target genes.

Table 2. List of genes targeted by miRNAs in cassava.

\begin{tabular}{|c|c|c|c|c|c|}
\hline miRNA & $\begin{array}{l}\text { Total } \\
\text { targets }\end{array}$ & $\begin{array}{l}\text { No. and name of } \\
\text { conserved targets }\end{array}$ & \multicolumn{3}{|c|}{ Conserved miRNA-targeted genes ID } \\
\hline $\operatorname{miR} 156$ & 36 & 7 SPLs & $\begin{array}{l}\text { Manes.01G026000; } \\
\text { Manes.12G009000; } \\
\text { Manes.13G011000 }\end{array}$ & $\begin{array}{l}\text { Manes.05G050800; } \\
\text { Manes.12G010200; }\end{array}$ & $\begin{array}{l}\text { Manes.09G032800; } \\
\text { Manes.13G009400; }\end{array}$ \\
\hline $\operatorname{miR} 159$ & 7 & $5 \mathrm{MYBs}$ & $\begin{array}{l}\text { Manes.01G175900; } \\
\text { Manes.05G052300; N }\end{array}$ & $\begin{array}{l}\text { Manes.02G133900; } \\
\text { 11G009900 }\end{array}$ & Manes.04G153700; \\
\hline $\operatorname{miR} 160$ & 9 & 7 ARFs & $\begin{array}{l}\text { Manes.04G019800; } \\
\text { Manes.08G007600; } \\
\text { Manes.18G072400 }\end{array}$ & $\begin{array}{l}\text { Manes.07G099400; } \\
\text { Manes.10G046700; }\end{array}$ & $\begin{array}{l}\text { Manes.07G099500; } \\
\text { Manes.11G146500; }\end{array}$ \\
\hline $\operatorname{miR} 164$ & 6 & 5 NACs & $\begin{array}{l}\text { Manes.02G047800; } \\
\text { Manes.14G080400; N }\end{array}$ & $\begin{array}{l}\text { Manes.03G105700; } \\
\text { 15G089700 }\end{array}$ & Manes.06G090500; \\
\hline miR166 & 20 & 7 HD-ZIPs & $\begin{array}{l}\text { Manes.03G059200; } \\
\text { Manes.11G034600; } \\
\text { Manes.16G074600 }\end{array}$ & $\begin{array}{l}\text { Manes.04G020100; } \\
\text { Manes.11G146000; }\end{array}$ & $\begin{array}{l}\text { Manes.04G133100; } \\
\text { Manes.14G122900; }\end{array}$ \\
\hline miR168 & 1 & $1 \mathrm{AGO}$ & Manes.02G219700 & & \\
\hline $\operatorname{miR} 171$ & 2 & $2 \mathrm{SCLS}$ & Manes.02G038400; N & 05G048700 & \\
\hline $\operatorname{miR} 172$ & 17 & $6 \mathrm{AP} 2 \mathrm{~s}$ & $\begin{array}{l}\text { Manes.04G027000; } \\
\text { Manes.11G139300; N }\end{array}$ & $\begin{array}{l}\text { Manes.07G101700; } \\
\text { 12G106400; Manes.1 }\end{array}$ & $\begin{array}{l}\text { Manes.09G080100; } \\
700\end{array}$ \\
\hline $\operatorname{miR319}$ & 4 & 3 TCPs & Manes.13G138300; N & 14G058400; Manes.15 & 000 \\
\hline $\operatorname{miR393}$ & 4 & 2 TIRs; 2 AFBs & $\begin{array}{l}\text { Manes.01G214300; } \\
\text { Manes.11G078600 }\end{array}$ & Manes.05G067800; & Manes.04G091500; \\
\hline miR394 & 2 & 2 F-boxes & Manes.12G010000; N & 13G010700 & \\
\hline miR395 & 4 & 1 APS & Manes.15G033600 & & \\
\hline $\operatorname{miR396}$ & 20 & 11 GRFs & $\begin{array}{l}\text { Manes.01G041800; } \\
\text { Manes.03G039500; } \\
\text { Manes.05G190700; } \\
\text { Manes.16G096400; N }\end{array}$ & $\begin{array}{l}\text { Manes.01G264700; } \\
\text { Manes.04G144700; } \\
\text { Manes.08G160800; } \\
\text { 18G049600 }\end{array}$ & $\begin{array}{l}\text { Manes.02G031200; } \\
\text { Manes.05G18390; } \\
\text { Manes.09G129700; }\end{array}$ \\
\hline
\end{tabular}



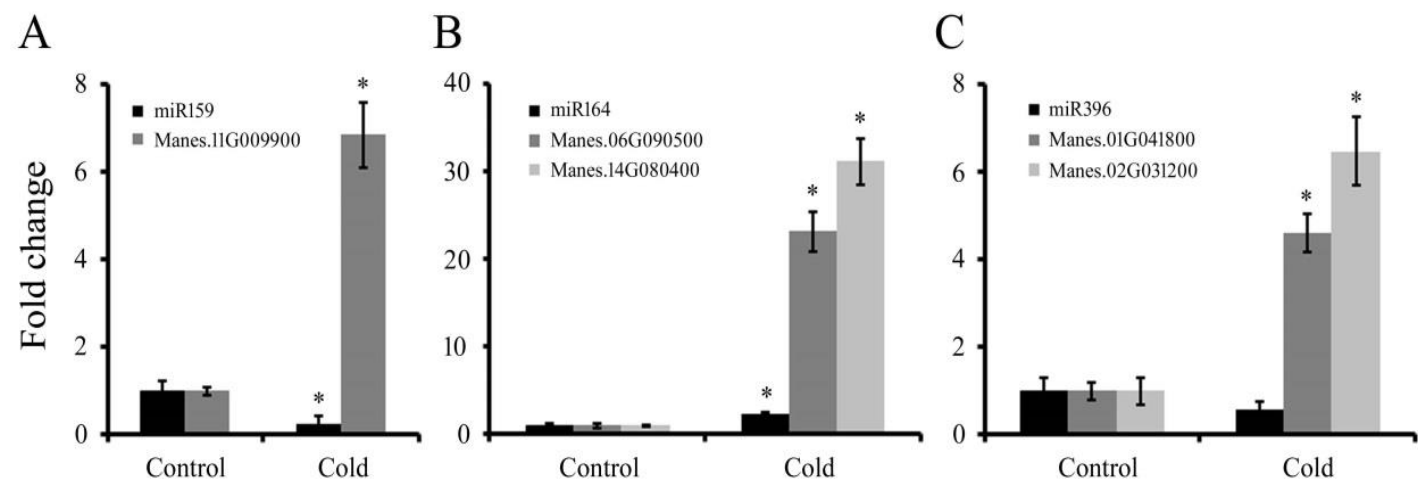

Fig 2. The expression patterns of three miRNAs and their targets under cold stress in cassava. U6 and MeACTIN were used as an internal control for miRNA and targets, respectively. Error bars represent \pm SD from three independent experiments. Asterisks indicate differences of statistical significance between CK and Cold samples plants $\left({ }^{*} p<0.05\right)$

Table 3. Differential expressed miRNA targets in response to cold stress.

\begin{tabular}{|c|c|c|c|}
\hline miRNA & $\begin{array}{l}\text { miRNA-targeted gene } \\
\text { ID }\end{array}$ & $\begin{array}{l}\text { Fold } \\
\text { change }\end{array}$ & Full name of miRNA-targeted genes \\
\hline \multirow[t]{5}{*}{ miR156 } & Manes.01G026000 & -2.86 & Squamosa promoter-binding-like protein 6 \\
\hline & Manes.02G118800 & 5.23 & Zinc finger domain-containing protein 66 \\
\hline & Manes.06G088300 & 4.55 & DUF863 domain-containing protein \\
\hline & Manes.08G081400 & 4.18 & E3 ubiquitin-protein ligase RDUF1-like \\
\hline & Manes.10G052500 & 2.39 & NAC transcription factors 5 \\
\hline miR159 & Manes.11G009900 & 4.76 & Transcription factor GAMYB-like \\
\hline \multirow[t]{2}{*}{$\operatorname{miR} 160$} & Manes.07G099500 & -2.86 & Auxin response factor 16 \\
\hline & Manes.18G072400 & 2.06 & Auxin response factor 17 \\
\hline \multirow[t]{2}{*}{ miR164 } & Manes.06G090500 & 5.68 & NAC transcription factors 17 \\
\hline & Manes.14G080400 & 13.68 & NAC transcription factors 62 \\
\hline \multirow[t]{3}{*}{ miR166 } & Manes.04G139200 & 3.08 & Beta-galactosidase 1 \\
\hline & Manes.06G159400 & 2.08 & Glutamate synthase 1 \\
\hline & Manes.18G105400 & 4.56 & Patellin-3 \\
\hline miR167 & Manes.06G167300 & -2.13 & Alpha-L-arabinofuranosidase $B$ \\
\hline \multirow[t]{4}{*}{ miR172 } & Manes.04G054500 & 2.29 & Transcription factor bHLH128 \\
\hline & Manes.07G034600 & -2.71 & $A B C$ transporter family protein \\
\hline & Manes.09G080100 & 2.26 & Floral homeotic protein APETALA 2 \\
\hline & Manes.S065500 & 2.05 & B-cell receptor-associated protein 31 \\
\hline miR319 & Manes.15G091000 & -2.38 & Transcription factor TCP3-like \\
\hline miR394 & Manes.13G010700 & 2.19 & F-box only protein 6 \\
\hline \multirow[t]{2}{*}{ miR395 } & Manes.12G129400 & -2.56 & Probable glucan 1,3-beta-glucosidase A \\
\hline & Manes.17G046600 & 3.54 & VQ motif-containing protein 4 \\
\hline \multirow[t]{8}{*}{ miR396 } & Manes.01G041800 & 2.03 & Growth-regulating factor 7 \\
\hline & Manes.02G031200 & 5.55 & Growth-regulating factor 10 \\
\hline & Manes.03G039500 & -2.44 & Growth-regulating factor 1 \\
\hline & Manes.07G044100 & 2.1 & NBS-LRR resistance protein RGH1 \\
\hline & Manes.07G044300 & 4.55 & NBS-LRR resistance protein $\mathrm{RGH} 2$ \\
\hline & Manes.07G044700 & 7.27 & NBS-LRR resistance protein RGH1 \\
\hline & Manes.07G045400 & 3.13 & NBS-LRR resistance protein RGH1 \\
\hline & Manes.14G115700 & -27.1 & Hypothetical protein \\
\hline miR477 & Manes.02G097400 & 2.16 & Disease resistance RPP13-like protein 4 \\
\hline
\end{tabular}

MiR164 showed slightly up-regulated expression in response to cold stress in this study, which was consistent with previous research (Li et al., 2017b). The miR396 and GRF regulatory network is evolutionarily conserved among plant species. The GRFs is a plant specific family of transcription factors defined by the presence of the WRC and QLQ protein domains (Ercoli et al., 2016). Seven out of the 9 Arabidopsis GRFs have a binding site for microRNA (miRNA) miR396 (Rodriguez et al.,
2010; Wang et al., 2011). GRFs have been implicated in the development and growth of plant organs and structures (Das Gupta and Nath, 2015). Previous studies have revealed that miR396 is responsive to a variety of environmental stresses, such as salt, drought, oxidative stress and cold stress (Liu et al., 2008; Zhou et al., 2010; Gao et al., 2015). Transgenic rice and Arabidopsis plants constitutively over-expressing osa-MIR396c showed reduced salt stress tolerance compared to that of 
wild-type plants (Gao et al., 2010). Our findings indicate that the expression of miR396 decreased significantly and its target genes was up-regulated during cold stress. To date, investigations on cassava miR396 and their targets are rather rare. Clarifying the biological functions of the miR396 targets in response to abiotic stresses will help uncover the function of miR396 in crops.

\section{Materials and methods}

\section{Plant material and stress treatment}

Cassava (Manihot esculenta) cultivar (60444) was used in the study. As previously described (Li et al., 2017a; Li et al., 2017b), the stems about $1.5 \mathrm{~cm}$ in size with one bud were cut and planted in MS plate for 2 weeks in a growth chamber at $26 \pm$ $2^{\circ} \mathrm{C}$, with a photoperiod of $16 \mathrm{~h}$ light and $8 \mathrm{~h}$ dark. For cold treatment, plants with a uniform growth status were treated at $4^{\circ} \mathrm{C}$ for $24 \mathrm{~h}$ in a chamber under $16 \mathrm{~h}$ light and $8 \mathrm{~h}$ dark. The youngest leaves and shoot apex were collected and then were immediately frozen in liquid nitrogen for RNA extraction and RNA sequencing. This protocol was chosen for two reasons: (i) we measure the expression of cold stress marker genes (known $D R E B / C B F s$ and CORs/RD29) using RNA derived from samples exposed to cold stress at $4^{\circ} \mathrm{C}$ for different time points, the expression of the marker genes is maximal at 24 hour after cold stress (Li et al., 2017b). (ii) We observed visible leave wilting in cold stress treatments. Untreated plants at the same stage grown in chamber under $16 \mathrm{~h}$ light and $8 \mathrm{~h}$ dark were used as controls. More than ten plants were harvested and pooled for each sample, and the collection was repeated three times as biological replicates.

\section{Library construction of degradome-seq and bioinformatic analysis}

Two degradome libraries (Control and Cold), one replicate for each library, were constructed as described previously with minor modifications (German et al., 2009). The total RNA extraction, libraries construction and deep sequencing were performed by the Guangzhou Genedenovo Biotechnology Co., Ltd (Guangzhou, China). First, poly (A) + mRNA were enriched by Oligo (dT) magnetic beads. Then, the cleavage products with a free $5^{\prime}$-monophosphate at their $3^{\prime}$ termini were ligated with 5' RNA adapters, followed with reverse transcription and PCR. Two libraries were sequenced on an Illumina Hiseq 2000 platform. The raw reads were pre-processed to filter low-quality reads, adaptors, tRNAs, rRNAs, snRNAs and snoRNAs. To identify genes targeted by miRNAs, the remaining clean reads were mapped to the reference genes (cDNA) of cassava by SOAP2 tools (Hurgobin, 2016), and the miRNA-mRNA pairs were searched and p-values were calculated using PAREsnip (Folkes et al., 2012). Additionally, the CleaveLand program was run to detect potentially cleaved miRNA targets (Addo-Quaye et al., 2009). All alignments with p-value $<0.05$ that possessed the $5^{\prime}$ end of the degradome sequence coincident with the 10th and 11th nucleotides were considered as miRNA targets.

\section{Functional annotations of the miRNA targets}

To investigate the putative biological functions of target genes and biological processes possibly regulated by miRNAs in cassava, Gene Ontology (GO) analysis were employed to annotate and classify target genes (Young et al., 2010). The GO categorization results were listed as three independent hierarchies for biological process, cellular component, and molecular function.

\section{Detection of miRNA and their targets using qRT-PCR}

Total RNA from the same samples was prepared using the RNA Plant kit (OMEGA). For miRNA, approximately $3 \mu \mathrm{g}$ of total RNA was reverse-transcribed using miRNA-specific stem-loop primers in a $20 \mu \mathrm{L}$ of reaction volume using a Fermentas RevertAid First trand cDNA Synthesis kit (Fermentas, USA). For miRNAs' targets, the cDNA was generated using $2 \mu \mathrm{g}$ of total RNA and OligodT18 primer with the PrimeScriptTM RT reagent kit (Takara). Real time qPCR assays were performed using the SYBR Premix ExTaq ${ }^{\text {TM }}$ (Takara) followed the manuals from the manufacturers. U6 small nuclear RNA and MeACTIN gene were used as the internal control for miRNA and corresponding targets, respectively. The expression level of the miRNAs and their targets in different samples were calculated by comparative $2^{-\triangle \Delta^{C T}}$ method. Standard deviations were calculated from three biological replicates. The primers used for qRT-PCR analysis are listed in Supplementary Table 2.

\section{Data access}

The degradome RNA sequencing raw data has been deposited in the NCBI Sequence Read Archive (SRA) under the BioProject accession number SRP133534.

\section{Conclusion}

This is the first comprehensive identification of cold-responsive miRNAs targets in cassava by degradome sequencing. A total of 151 miRNA-target pairs were identified, including many new target miRNA pairs. This study provides important data for better understanding the molecular mechanisms of resistance to cold in cassava. Further experiments on the identified target genes are necessary to explore potential mechanisms of abiotic stress regulation in cassava.

\section{Acknowledgments}

We thank Dr. Xueyi Tian for helpful comments on this manuscript. This work was supported by the National Natural Science Foundation of China (31701484, 31561143012 and 31701903), the National Key R\&D Program of China (2018YFD1000500), and the grants from the Young Elite Scientists Sponsorship Program by CSTC (CSTC-QN201702).

\section{Supplementary data}

Supplementary Table S1. Detailed information of the miRNA targets identified from cassava.

Supplementary Table S2. Summary of primers used in this study.

\section{References}

Addo-Quaye C, Miller W, Axtell MJ (2009) CleaveLand: a pipeline for using degradome data to find cleaved small RNA 
targets Bioinformatics. 25, 130-131

An D, Yang J, Zhang P (2012) Transcriptome profiling of low temperature-treated cassava apical shoots showed dynamic responses of tropical plant to cold stress BMC Genomics. 13, 64.

An D, Ma Q, Wang H, Yang J, Zhou W, Zhang P (2017) Cassava $\mathrm{C}$-repeat binding factor 1 gene responds to low temperature and enhances cold tolerance when overexpressed in Arabidopsis and cassava Plant Molecular Biology 94, 109-124.

An F, Li G, Li QX, Li K, Carvalho LJ, Ou W, Chen S (2016) The comparatively proteomic analysis in response to cold stress in cassava plantlets Plant Mol Biol Report. 34, 1095-1110.

Ballen-Taborda C, Plata G, Ayling S, Rodriguez-Zapata F, Becerra Lopez-Lavalle LA, Duitama J, Tohme J (2013) Identification of cassava microRNAs under abiotic stress. International J Genomics. 857-986.

Bresso EG, Chorostecki U, Rodriguez RE, Palatnik JF, and Schommer C (2018) Spatial control of gene expression by miR319-Regulated TCP transcription factors in leaf development. Plant physiology. 176, 1694-1708.

Chen X, Xia J, Xia Z, Zhang H, Zeng C, Lu C, Zhang W, Wang W (2015) Potential functions of microRNAs in starch metabolism and development revealed by miRNA transcriptome profiling of cassava cultivars and their wild progenitor. BMC plant biology. 15, 33.

Cui LG, Shan JX, Shi M, Gao JP, Lin HX (2014) The miR156-SPL9-DFR pathway coordinates the relationship between development and abiotic stress tolerance in plants. Plant Journal: for Cell and Molecular Biology. 80, 1108-1117.

Das Gupta M, Nath U (2015) Divergence in patterns of leaf growth polarity is associated with the expression divergence of miR396. The Plant Cell. 27, 2785-2799.

E1-Sharkawy MA (2004) Cassava biology and physiology. Plant Molecular Biology. 56, 481-501.

Eckardt NA (2012) A microRNA cascade in plant defense. The Plant Cell. 24, 840.

Eldem V, Celikkol Akcay U, Ozhuner E, Bakir Y, Uranbey S, Unver T (2012) Genome-wide identification of miRNAs responsive to drought in peach (Prunus persica) by high-throughput deep sequencing. PloS one. 7, e50298

Ercoli MF, Rojas AM, Debernardi JM, Palatnik JF, Rodriguez RE (2016) Control of cell proliferation and elongation by miR396. Plant Signaling \& Behavior. 11, e1184809.

Fang Y, Xie K, Xiong L (2014) Conserved miR164-targeted NAC genes negatively regulate drought resistance in rice. Journal of Experimental Botany. 65, 2119-2135.

Feng K, Nie X, Cui L, Deng P, Wang M, Song W (2017) Genome-wide identification and characterization of salinity stress-responsive miRNAs in wild emmer wheat (Triticum turgidum ssp. dicoccoides) Genes (Basel). 6:8(6). pii: E156 8.

Folkes L, Moxon S, Woolfenden HC, Stocks MB, Szittya G, Dalmay T, Moulton V (2012) PAREsnip: a tool for rapid genome-wide discovery of small RNA/target interactions evidenced through degradome sequencing. Nucleic Acids Research. 40, e103.

Gao F, Wang K, Liu Y, Chen Y, Chen P, Shi Z, Luo J, Jiang D, Fan F, Zhu Y, Li S (2015) Blocking miR396 increases rice yield by shaping inflorescence architecture. Nature Plants. 2, 15196.

Gao P, Bai X, Yang L, Lv D, Li Y, Cai H, Ji W, Guo D, Zhu Y (2010) Over-expression of osa-MIR396c decreases salt and alkali stress tolerance. Planta. 231, 991-1001.
Geng M, Li H, Jin C, Liu Q, Chen C, Song W, Wang C (2014) Genome-wide identification and characterization of miRNAs in the hypocotyl and cotyledon of cauliflower (Brassica oleracea $L$ var botrytis) seedlings. Planta. 239, 341-356.

German MA, Luo S, Schroth G, Meyers BC, Green PJ (2009) Construction of Parallel Analysis of RNA Ends (PARE) libraries for the study of cleaved miRNA targets and the RNA degradome. Nature Protocols. 4, 356-362.

Guo HS, Xie Q, Fei JF, Chua NH (2005) MicroRNA directs mRNA cleavage of the transcription factor NAC1 to downregulate auxin signals for Arabidopsis lateral root development. The Plant Cell. 17, 1376-1386.

Guo N, Zhang Y, Sun X, Fan H, Gao J, Chao Y, Liu A, Yu X, Cai Y, Lin $Y$ (2018) Genome-wide identification of differentially expressed miRNAs induced by ethephon treatment in abscission layer cells of cotton (Gossypium hirsutum). Gene. 676, 263-268.

Hake S (2003) MicroRNAs: a role in plant development Current biology: CB. 13, R851-852.

Huang L, Ye Z, Bell RW, Dell B (2005) Boron nutrition and chilling tolerance of warm climate crop species. Annals of Botany. 96, 755-767.

Hurgobin B (2016) Short read alignment using SOAP2. Methods in Molecular Biology. 1374, 241-252.

Jones-Rhoades MW, Bartel DP (2004) Computational identification of plant microRNAs and their targets, including a stress-induced miRNA. Molecular Cell. 14, 787-799.

Kim JH, Woo HR, Kim J, Lim PO, Lee IC, Choi SH, Hwang D, Nam HG (2009) Trifurcate feed-forward regulation of age-dependent cell death involving miR164 in Arabidopsis. Science. 323, 1053-1057.

Koyama T, Mitsuda N, Seki M, Shinozaki K, Ohme-Takagi M (2010) TCP transcription factors regulate the activities of ASYMMETRIC LEAVES1 and miR164, as well as the auxin response, during differentiation of leaves in Arabidopsis. The Plant Cell. 22, 3574-3588.

Laufs P, Peaucelle A, Morin H, Traas J (2004) MicroRNA regulation of the CUC genes is required for boundary size control in Arabidopsis meristems. Development. 131, 4311-4322.

Li J, Guo G, Guo W, Guo G, Tong D, Ni Z, Sun Q, Yao Y (2012) miRNA164-directed cleavage of ZmNAC1 confers lateral root development in maize (Zea mays L). BMC Plant Biology. 12, 220.

Li S, Yu X, Cheng Z, Yu X, Ruan M, Li W, Peng M (2017a) Global gene expression analysis reveals crosstalk between response mechanisms to cold and drought stresses in cassava seedlings. Frontiers in Plant Science. 8, 1259.

Li S, Yu X, Lei N, Cheng Z, Zhao P, He Y, Wang W, Peng M (2017b) Genome-wide identification and functional prediction of cold and/or drought-responsive IncRNAs in cassava. Scientific Reports. 7, 45981.

Li X, Bian H, Song D, Ma S, Han N, Wang J, Zhu M (2013a) Flowering time control in ornamental gloxinia (Sinningia speciosa) by manipulation of miR159 expression. Annals of Botany. 111, 791-799.

Li Y, Alonso-Peral M, Wong G, Wang MB, Millar AA (2016) Ubiquitous miR159 repression of MYB33/65 in Arabidopsis rosettes is robust and is not perturbed by a wide range of stresses. BMC Plant Biology. 16, 179.

Li Z, Peng J, Wen X, Guo H (2013b) Ethylene-insensitive3 is a senescence-associated gene that accelerates age-dependent leaf senescence by directly repressing miR164 transcription 
in Arabidopsis. The Plant Cell. 25, 3311-3328.

Liu, HH, Tian, X, Li, YJ, Wu, CA, and Zheng, CC (2008) Microarray-based analysis of stress-regulated microRNAs in Arabidopsis thaliana Rna 14, 836-843

Lu S, Sun YH, Chiang VL (2008) Stress-responsive microRNAs in Populus. The Plant Journal: for Cell and Molecular Biology. 55, 131-151.

Lv DK, Bai X, Li Y, Ding XD, Ge Y, Cai H, Ji W, Wu N, Zhu YM (2010) Profiling of cold-stress-responsive miRNAs in rice by microarrays. Gene. 459, 39-47.

Ori N, Cohen AR, Etzioni A, Brand A, Yanai O, Shleizer S, Menda $\mathrm{N}$, Amsellem Z, Efroni I, Pekker I, Alvarez JP, Blum E, Zamir D, and Eshed $Y$ (2007) Regulation of LANCEOLATE by miR319 is required for compound-leaf development in tomato. Nature Genetics. 39, 787-791.

Raman S, Greb T, Peaucelle A, Blein T, Laufs P, Theres K (2008) Interplay of miR164, CUP-SHAPED COTYLEDON genes and LATERAL SUPPRESSOR controls axillary meristem formation in Arabidopsis thaliana. The Plant Journal: for Cell and Molecular Biology. 55, 65-76.

Reyes JL, Chua NH (2007) ABA induction of miR159 controls transcript levels of two MYB factors during Arabidopsis seed germination. The Plant journal: for Cell and Molecular Biology. 49, 592-606.

Rodriguez RE, Mecchia MA, Debernardi JM, Schommer C, Weigel D, Palatnik JF (2010) Control of cell proliferation in Arabidopsis thaliana by microRNA miR396. Development. 137, 103-112.

Rogers K, Chen X (2013) Biogenesis, turnover, and mode of action of plant microRNAs. The Plant Cell. 25, 2383-2399.

Sahu S, Rao AR, Pandey J, Gaikwad K, Ghoshal S, and Mohapatra T (2018) Genome-wide identification and characterization of IncRNAs and miRNAs in cluster bean (Cyamopsis tetragonoloba). Gene. 667, 112-121.

Samad AFA, Sajad M, Nazaruddin N, Fauzi IA, Murad AMA, Zainal Z, Ismail I (2017) MicroRNA and transcription factor: key players in plant regulatory network. Frontiers in Plant Science. 8, 565.

Shriram V, Kumar V, Devarumath RM, Khare TS, Wani SH (2016) MicroRNAs as potential targets for abiotic stress tolerance in plants. Frontiers in Plant Science. 7, 817.

Thiebaut F, Rojas CA, Almeida KL, Grativol C, Domiciano GC, Lamb CR, Engler Jde A, Hemerly AS, Ferreira PC (2012) Regulation of miR319 during cold stress in sugarcane. Plant, Cell \& Environment. 35, 502-512.

Wang L, Gu X, Xu D, Wang W, Wang H, Zeng M, Chang Z, Huang $H$, Cui X (2011) miR396-targeted AtGRF transcription factors are required for coordination of cell division and differentiation during leaf development in Arabidopsis. Journal of Experimental Botany. 62, 761-773.

Willmann MR, Poethig RS (2007) Conservation and evolution of miRNA regulatory programs in plant development. Current Opinion in Plant Biology. 10, 503-511.

Xu J, Duan X, Yang J, Beeching JR, Zhang P (2013) Coupled expression of $\mathrm{Cu} / \mathrm{Zn}$-superoxide dismutase and catalase in cassava improves tolerance against cold and drought stresses. Plant Signaling \& Behavior. 8, e24525.

Xue T, Liu Z, Dai X, Xiang F (2017) Primary root growth in Arabidopsis thaliana is inhibited by the miR159 mediated repression of MYB33, MYB65 and MYB101. Plant Science: an International Journal of Experimental Plant Biology. 262, 182-189.
Yadav A, Khan Y, Prasad M (2016) Dehydration-responsive miRNAs in foxtail millet: genome-wide identification, characterization and expression profiling. Planta. 243, 749-766.

Yang C, Li D, Mao D, Liu X, Ji C, Li X, Zhao X, Cheng Z, Chen C, Zhu $L$ (2013) Overexpression of microRNA319 impacts leaf morphogenesis and leads to enhanced cold tolerance in rice (Oryza sativa L). Plant, Cell \& Environment. 36, 2207-2218.

Young MD, Wakefield MJ, Smyth GK, Oshlack A (2010) Gene ontology analysis for RNA-seq: accounting for selection bias. Genome Biology. 11, R14.

Zeng C, Wang W, Zheng Y, Chen X, Bo W, Song S, Zhang W, Peng $M$ (2010) Conservation and divergence of microRNAs and their functions in Euphorbiaceous plants. Nucleic Acids Research. 38, 981-995.

Zeng C, Ding Z, Zhou F, Zhou Y, Yang R, Yang Z, Wang W, Peng M (2017) The discrepant and similar responses of genome-wide transcriptional profiles between drought and cold stresses in cassava. International Journal of Molecular Sciences. 12:18(12). pii: E2668.

Zeng $C$, Chen Z, Xia J, Zhang K, Chen X, Zhou Y, Bo W, Song S, Deng D, Guo X, Wang B, Zhou J, Peng H, Wang W, Peng M, and Zhang W (2014) Chilling acclimation provides immunity to stress by altering regulatory networks and inducing genes with protective functions in cassava. BMC Plant Biology. 14, 207.

Zhang B, Pan X, Cobb GP, Anderson TA (2006) Plant microRNA: a small regulatory molecule with big impact. Developmental Biology. 289, 3-16.

Zhang $H$, Yin L, Wang $H$, Wang $G$, Ma X, Li M, Wu H, Fu Q, Zhang Y, Yi H (2017) Genome-wide identification of Hami melon miRNAs with putative roles during fruit development. PloS one. 12, e0180600.

Zhang J, Wei L, Jiang J, Mason AS, Li H, Cui C, Chai L, Zheng B, Zhu Y, Xia Q, Jiang L, Fu D (2018) Genome-wide identification, putative functionality and interactions between IncRNAs and miRNAs in Brassica species. Scientific Reports. 8, 4960.

Zhao JP, Jiang XL, Zhang BY, Su XH (2012) Involvement of microRNA-mediated gene expression regulation in the pathological development of stem canker disease in Populus trichocarpa. PloS one 7. e44968.

Zhao Z, Xue Y, Yang H, Li H, Sun G, Zhao X, Ding D, Tang J (2016) Genome-Wide Identification of miRNAs and Their targets involved in the developing internodes under maize ears by responding to hormone signaling. PloS one. 11, e0164026.

Zhou L, Liu Y, Liu Z, Kong D, Duan M, Luo L (2010) Genome-wide identification and analysis of drought-responsive microRNAs in Oryza sativa. Journal of Experimental Botany. 61, 4157-4168.

Zhou M, Li, D Li, Z Hu, Q Yang C, Zhu L, Luo H (2013) Constitutive expression of a miR319 gene alters plant development and enhances salt and drought tolerance in transgenic creeping bentgrass Plant physiology. 161, 1375-1391. 